\title{
Barbituric Acid Tautomers: DFT Computations of Keto- Enol Conversions, Frontier Molecular Orbitals and Quadrupole Coupling Constants
}

\author{
Kun Harismah ${ }^{1, *(\mathbb{D})}$, Farzaneh Fazeli ${ }^{2}{ }^{(\mathbb{D})}$, Hasan Zandi ${ }^{3(\mathbb{D})}$ \\ 1 Department of Chemical Engineering, Faculty of Engineering, Universitas Muhammadiyah Surakarta, Surakarta, \\ Indonesia; kun.harismah@ums.ac.id (K.H.); \\ 2 Department of Biology, Payame Noor University, Tehran, Iran; seacorales@yahoo.com (F.F.); \\ 3 Department of Chemistry, Faculty of Science, University of Qom, Qom, Iran; hasan_zandi@ yahoo.com (H.Z.); \\ * Correspondence: kun.harismah@ums.ac.id;
}

Scopus Author ID 56982926300

Received: 26.02.2021; Revised: 30.03.2021; Accepted: 4.04.2021; Published: 20.04.2021

\begin{abstract}
In this work, density functional theory (DFT) computations were performed to investigate tautomeric formation processes of barbituric acid (BA). Ten tautomers were totally investigated for the purpose based on the movement of hydrogen atoms among nitrogen and oxygen atoms providing one pure keto form (BA1) and nine other keto-enol forms. The structures were optimized, and BA1 was found to be the most stable one, and both BA3 and BA7 were found to be the most unstable ones. The point was that the ring structure was broken for both BA3 and BA7, but the structure's stability was still approved. Indeed, such serious tautomeric conversion with breaking the structure warns for using such BA bio-organic molecules for further applications, especially in pharmacy-related ones, in which side effects or byproduct synthesis might appear. Further analyses of frontier molecular orbitals features indicated the effects of such tautomerism processes on all model systems, in which more details were obtained by atomic-scale quadrupole coupling constant (Qcc). All obtained results approved significant changes of tautomers regarding molecular and atomic scale features with more or less significant effects regarding the original BA1 reference model.
\end{abstract}

Keywords: barbituric acid; tautomer; frontier molecular orbital; NQR; DFT.

(C) 2021 by the authors. This article is an open-access article distributed under the terms and conditions of the Creative Commons Attribution (CC BY) license (https://creativecommons.org/licenses/by/4.0/).

\section{Introduction}

Soon after the pioneering work of Watson and Crick for the recognition of nucleic acid structures, several attempts have been dedicated to investigating different aspects of the building blocks of life [1-3]. The genes are very much important in living systems, and they could lead several important roles to maintain life [4]. Two types of purine and pyrimidine nucleobases are the main constructing members of nucleic acids, in which adenine (A) and guanine $(\mathrm{G})$ are purines, whereas cytosine $(\mathrm{C})$, thymine $(\mathrm{T})$, and uracil $(\mathrm{U})$ are pyrimidines [59]. In addition to original nucleobases, various synthetic forms have been produced for different purposes, with biomedical applications' most important ones [10]. In this regard, pharmaceutical compounds have arisen from nucleobase derivatives with very much compatibility with living systems and efficient medications [11]. Earlier works provided insightful information on such nucleobase-pharmacy-related compounds with different methodologies and targeted problems [12-15]. Besides the benefits of such nucleobase-derived pharmaceutical compounds, tautomerism has been seen to be taken place in such bio-organic 
molecules yielding different structures and corresponding properties [16]. Structure-activity relationship (SAR) is an important aspect for the efficacy of pharmaceutical compounds, in which changes of structures in tautomerism processes could lead to different activities somehow could be seen as side effects in the patients under medication [17]. Therefore, careful analysis of such tautomeric structures could help understand better structural features of pharmaceutical bio-organic compounds to decide on any required structural modifications [18]. Molecular scale computations are an important tool for achieving such a purpose, in which molecular and atomic descriptors could be very well evaluated for interpretation of the structural problems [19-22]. Within this work, such a tool was employed for careful analyses of tautomers of barbituric acid (BA), which is a starting compound for the synthesis of barbiturate drugs [23].

BA is a derivative of uracil nucleobase with a potency of contributing to the tautomerism process [24]. Although earlier works tried to describe various aspects of this bioorganic compound, such scientific explorations have not yet been finished [25-28]. There are two nitrogen atoms and three oxygen atoms in the BA structure composing a heterocyclic ring, in which the hydrogen atom can move among nitrogen and oxygen atomic sites to provide ketoenol tautomers for BA (Figure 1). In such processes, structural features and corresponding electronic environments could detect such hydrogen atom movement perturbation. To better clarify this hypothesis, all possible tautomers of BA were systematically investigated in this work to reach the purpose of molecular and atomic scale analyses of BA and tautomers. Density functional theory (DFT) quantum-chemical computations were performed to stabilize BA's possible tautomeric structures and evaluate their molecular and atomic descriptors (Tables 1 and 2, Figures $1-3$ ). Consequently, this work's major goal was to investigate tautomers of BA employing DFT computed results of molecular and atomic scales.
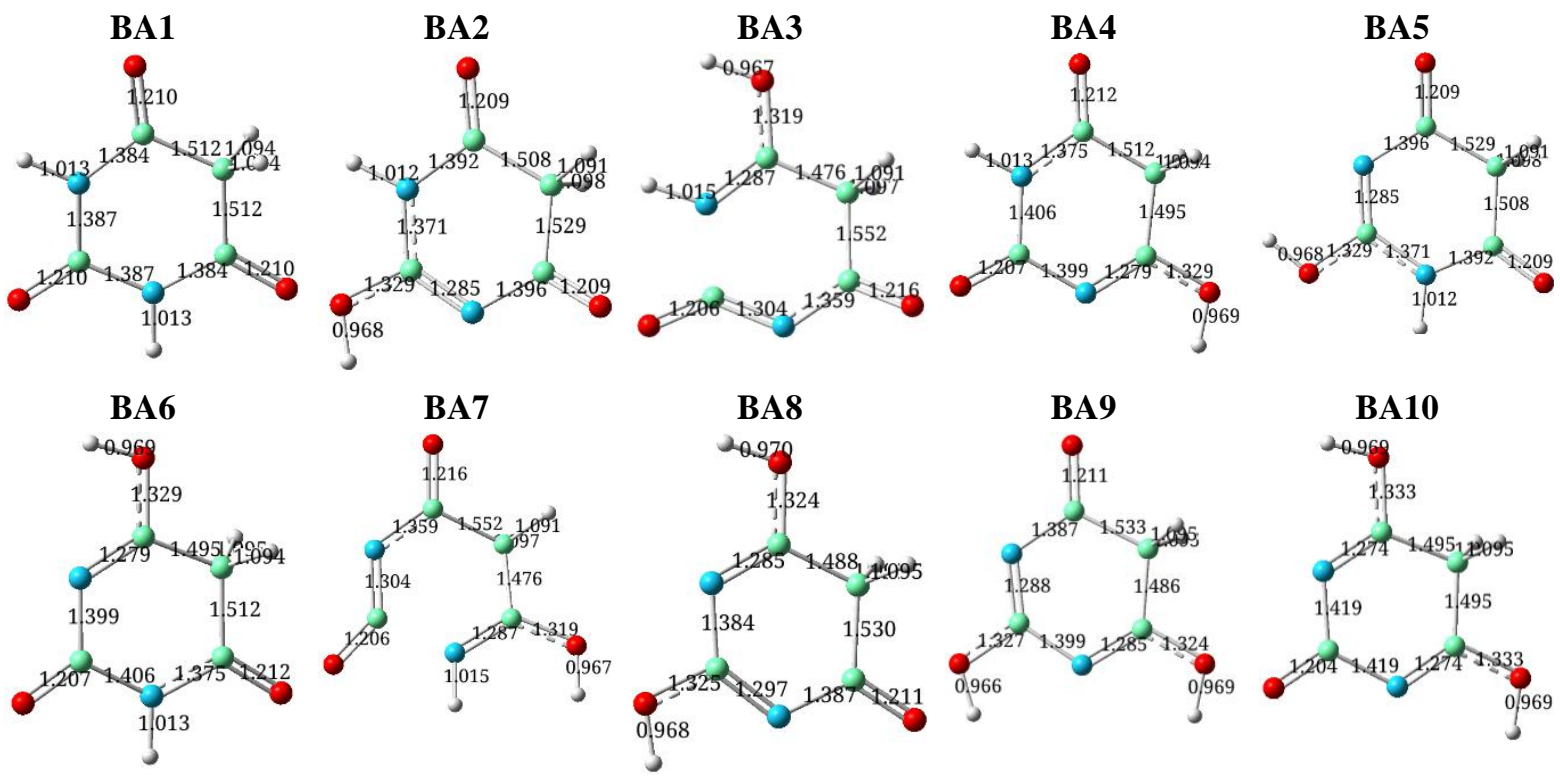

Figure 1. The optimized tautomers of BA, including bond distances $(\AA)$.

\section{Materials and Methods}

Quantum-chemical DFT computations were performed at the WB97XD/6-31+G** level of theory as implemented in the Gaussian program [29]. The original BA (C4H4N2O3) structure was obtained from the ChemSpider structural bank with ID 5976 [30]. Subsequently, movements of two hydrogen atoms of nitrogen atoms to other oxygen atomic sites of 
heterocycle were done to provide nine other tautomers, including keto-enol forms of BA, totally ten structures from BA1 to BA10 (Figure 1). Each structure was optimized to reach its minimum structure with further approval by frequency calculations examining any imaginary frequencies' exclusion. By performing these processes, quantities and qualities of molecular descriptors and visualization of infrared (IR) spectra were obtained for BA's ten molecular systems (Table 1 and Figures 1 - 3). In Figure 1, the optimized models of tautomers were presented in addition to their bond distances. In Figure 2, visualized IR spectra were shown. In Figure 3, frontier molecular orbitals representations, including distribution patterns of the highest occupied and the lowest unoccupied molecular orbitals (HOMO and LUMO) and electrostatic potential (ESP) surfaces, were shown. In Table 1, molecular descriptors were summarized, which included total energy (TE), total energy difference of a structure from the most stable structure $(\triangle T E)$, energy values of HOMO and LUMO, energy difference of HOMO and LUMO as energy gap (EG), energy average of HOMO and LUMO as Fermi energy (FE), dipole moment (DM) and volume (V). Moreover, related diagrams were also embedded in Table 1 to show the variations of parameters visually. To better describe the models in detail, quantities of atomic-scale quadrupole coupling constant (Qcc) were evaluated for nitrogen, oxygen, and hydrogen atoms of BA1 - BA10 to discuss the model systems by the variations of atomic electronic sites. In Table 2, quantities of Qcc were summarized in addition to related visual diagrams. Values of Qcc could show any perturbation to electronic sites of atoms to declare what has going on at the atomic scale in chemical systems [31-35]. Such Qcc parameters could be measured by nuclear quadrupole resonance (NQR) spectroscopy in experiments. They could be very well reproduced by quantum-chemical computations [36-38]. Hence, molecular and atomic scale descriptors were provided for discussing the main problem of this work on tautomerism of BA bio-organic molecule.

\section{Results and Discussion}

Ten possible tautomeric structures were found for BA through performing DFT-based optimization processes. As shown in Figure 1, BA3 and BA7 detected serious structural deformations by opening the ring bonds during tautomerism. Other tautomers showed that they could still maintain the ring system in such tautomerism processes. Only one tautomer, the original BA1 structure, was in pure keto form, and all other nine tautomers were in a mixture of keto-enol tautomers. As there were two hydrogen atoms with a potency of contributing to tautomerism processes for three oxygen atoms, pure enol form was a note found for the tautomers. Analyses of bond distances for such BA1 to BA10 structures could show that bond distances detected the effects of perturbations of tautomerism processes, especially for those bonds in the sites of tautomeric conversions. However, because of BA's small molecular size, variations of distances of other bonds far from the direct tautomerism sites were also meaningful. Such achievement could be very well seen by comparing the visualized IR spectra. The changes of spectrum from one tautomer to another one were significantly obvious. In such IR spectra, information of effects of tautomeric formations on vibrational frequencies and corresponding bond energies were detected for BA1-BA10 tautomers. Indeed, this is an important factor to be carefully considered for pharmaceutical-related compounds, especially for direct medications, in which possible formations of tautomers could change the structural features, and the already expected activated could be changed in this way. Therefore, it is useful to examine their various features through computer-based works to explore the systems at the lowest possible study scales. 


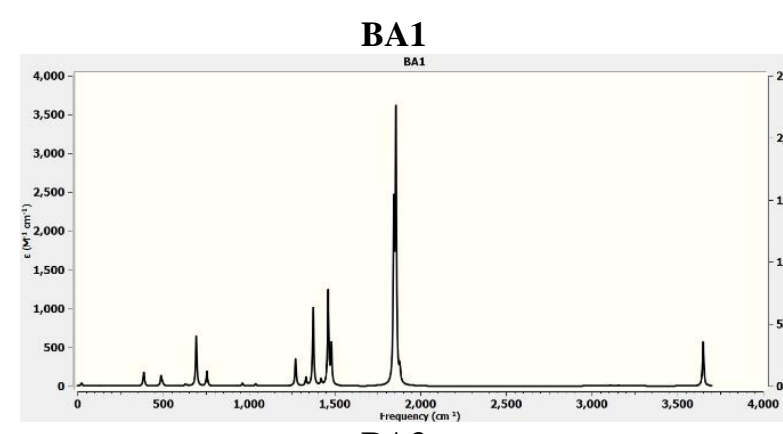

BA3

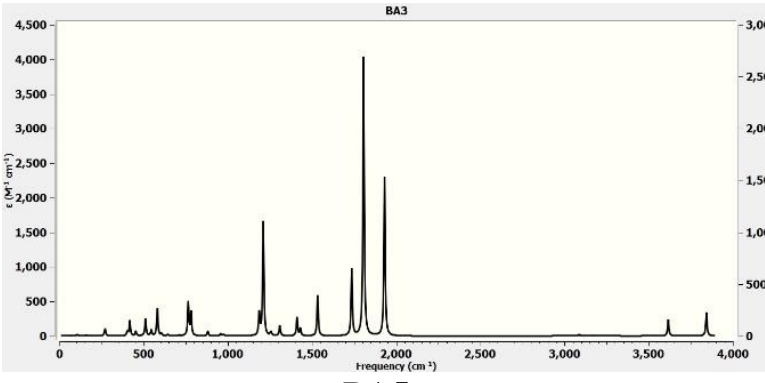

BA5

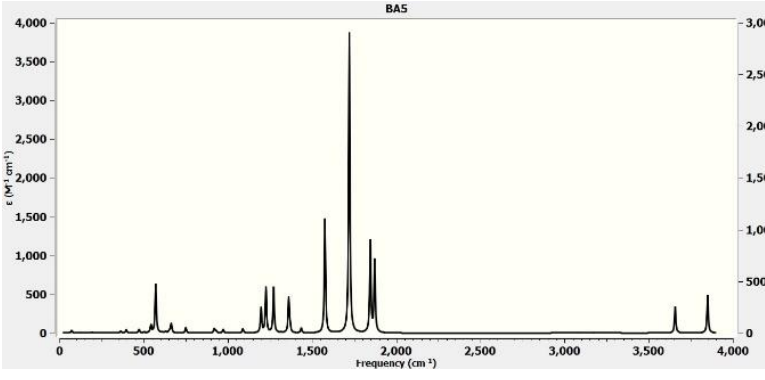

BA7

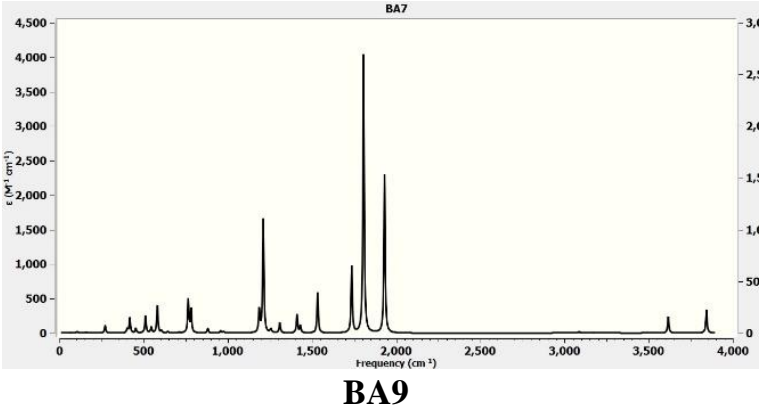

BA9

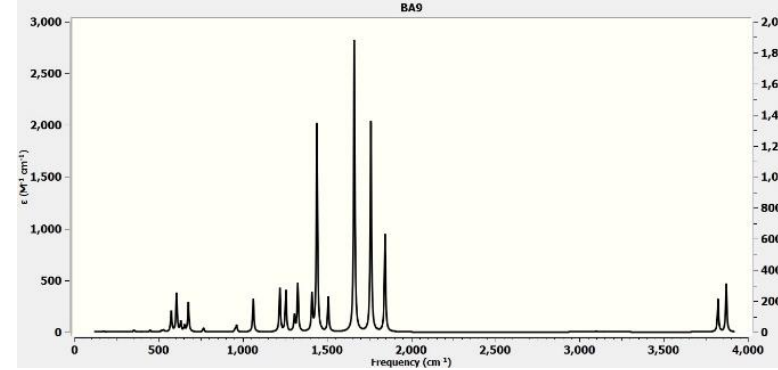

Figure 2. Visualized IR spectra for the models of BA.

BA2

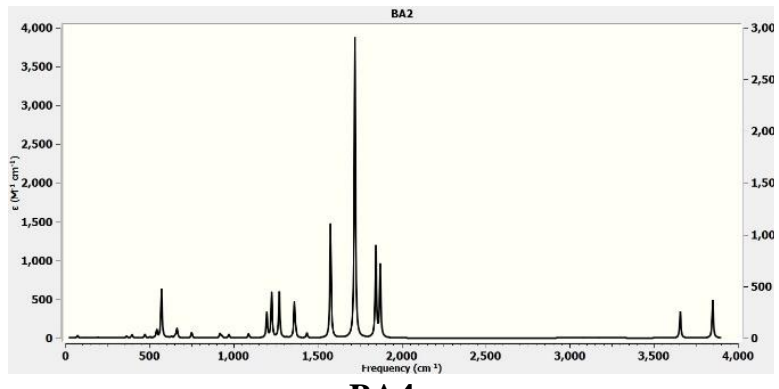

BA4

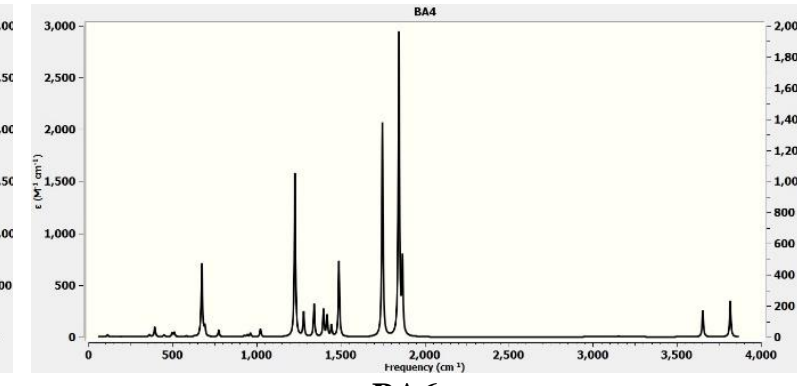

BA6

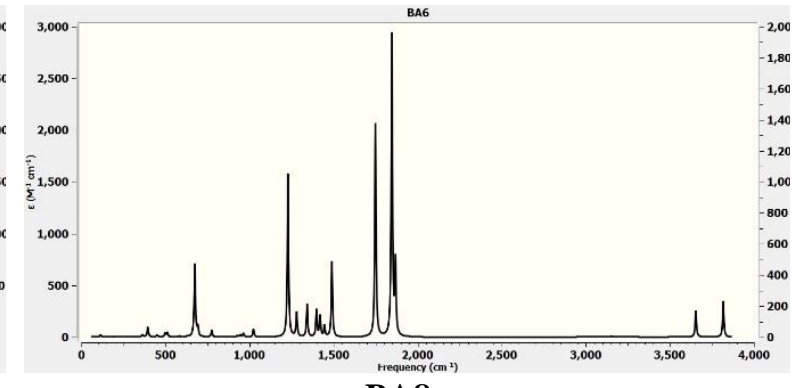

BA8

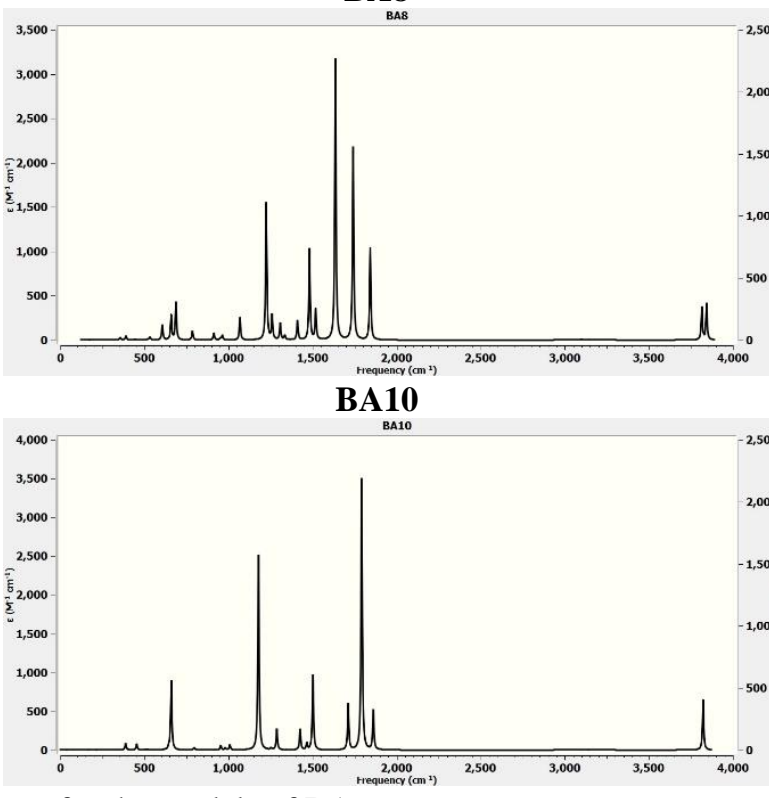

More results in detail were summarized in Table 1 and its related diagrams to discuss more about such important achievement. Comparing the TE results could show different stabilities for tautomers, with the most favorability for BA1 and the least favorability for both BA3 and BA7. Although the required energy for tautomeric conversion to those of the lowest stability ones was meaningful, several types of resources such as heat, weather temperature, or even sunshine might supply that energy for tautomeric conversion occurrence. Therefore, knowing such mechanisms at the lowest possible scales of computer-based works could 
provide insightful information for further investigations of bio-organic pharmacy-related compounds [39]. Formations of other tautomers were easier with low values of the required energy for tautomeric conversions showing the possibility of arising side effects for direct use of a substance or obtaining byproducts of its starting synthesis processes. Purity is an important parameter for synthetic materials, in which mixing such tautomers might increase impurity for obtaining a specified product.

Table 1. Molecuar descriptors and related diagrams. Energies are in eV, DM is in Debye and V is in $\mathrm{cm}^{3} / \mathrm{mol}^{\text {. }}$

\begin{tabular}{|c|c|c|c|c|c|c|c|c|}
\hline Model & TE & $\Delta \mathrm{TE}$ & HOMO & LUMO & EG & FE & DM & $\mathbf{V}$ \\
\hline BA1 & -13332.207 & 0 & -10.095 & 0.117 & 10.212 & -4.989 & 0.171 & 82.121 \\
\hline BA2 & -13331.336 & 0.869 & -9.711 & -0.047 & 9.664 & -4.879 & 3.722 & 65.631 \\
\hline BA3 & -13329.982 & 2.225 & -9.148 & -0.466 & 8.681 & -4.807 & 10.478 & 70.469 \\
\hline BA4 & -13331.529 & 0.679 & -10.149 & -0.111 & 10.038 & -5.130 & 3.498 & 78.643 \\
\hline BA5 & -13331.339 & 0.869 & -9.711 & -0.047 & 9.664 & -4.879 & 3.723 & 81.429 \\
\hline BA6 & -13331.529 & 0.679 & -10.149 & -0.111 & 10.038 & -5.130 & 3.498 & 72.402 \\
\hline BA7 & -13329.983 & 2.225 & -9.147 & -0.467 & 8.680 & -4.807 & 10.479 & 78.021 \\
\hline BA8 & -13330.816 & 1.392 & -9.588 & -0.377 & 9.211 & -4.982 & 4.045 & 77.893 \\
\hline BA9 & -13330.692 & 1.515 & -9.517 & -0.385 & 9.132 & -4.951 & 7.065 & 74.067 \\
\hline BA10 & -13330.732 & 1.475 & -9.850 & -0.047 & 9.803 & -4.949 & 5.404 & 90.183 \\
\hline 2.5 & \multicolumn{2}{|l|}{$\Delta \mathrm{TE}$ Values } & \multicolumn{3}{|c|}{ FMO Energies } & \\
\hline \multicolumn{3}{|l|}{2.5} & & \multicolumn{2}{|l|}{ IOMO =LUMO } & $\begin{array}{l}90 \\
80\end{array}$ & $\begin{array}{l}\text { DM and VV } \\
\text { DMM }\end{array}$ & \\
\hline \multirow{2}{*}{\multicolumn{9}{|c|}{1.5}} \\
\hline & & & & & & & & \\
\hline 1 & & & & $13 \beta^{B A}$ & $17 \beta^{A B} B$ & $\begin{array}{l}40 \\
30\end{array}$ & & \\
\hline 0.5 & & & -10 & & & $\begin{array}{c}20 \\
10 \\
0\end{array}$ & & . 1 \\
\hline
\end{tabular}

Further results of Table 1 were focused on frontier molecular orbitals features, including quantities of HOMO, LUMO, EG, and FE in addition to qualitative representations of HOMO and LUMO distribution patterns and ESP surfaces (Figure 3). The results indicated that such molecular orbitals features detected tautomerism effects, in which the effects were very much meant for BA3 and BA7, with the lowest stabilities among the tautomers. It is known that HOMO and LUMO are two important energy levels of molecular orbitals, in which electron-accepting and denoting or even electron transferring could be somehow led with the properties of these two molecular orbitals. Therefore, their quantities are important in addition to their distribution patterns showing the shapes of such molecular orbitals in qualitative mode. Among BA1 - BA10 tautomers, regarding the reference BA1 structure, HOMO and LUMO levels of all other tautomers detected the effects of hydrogen atom movements with the most significant effects for those of BA3 and BA7. Variations of such features could yield new electronic properties of the perturbated structure different from the original reference structure. Therefore, such mentioned side effects or byproduct synthesis might be achieved among the use of such small molecular bio-organic systems. As could be seen by the related diagram of Table 1, it could be seen that not only the levels of HOMO and LUMO but quantities of other EG and FA parameters could detect the effects of hydrogen atoms movements in the tautomeric formations. The shapes of orbitals in Figure 3 and ESP surfaces could show such deviation visually. Those of BA3 and BA7 were significantly changed in contrast with slight changes of other tautomers compared with the BA1 reverence model. It is important to mention here that properties for subunits of atoms and molecules are very important to assign overall electronic features for the structure, in which ESP surfaces could show such changes for the systems. The colors of red, yellow, green, and blue show ranges of electron environments from the most negative to the most positive sites. Variations of colors in the investigated systems could show 
such electronic nature changes for the model systems due to perturbations of tautomeric structure formations. Values of DM and V also showed changes of electric charge distribution at the molecular surfaces and resulting volume in the tautomeric structures, all approving the importance of careful analysis of such bio-organic molecules.

BA1

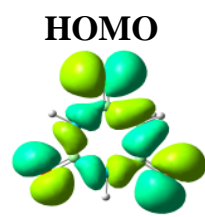

BA3

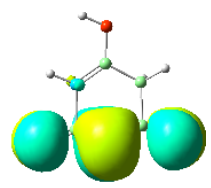

BA5

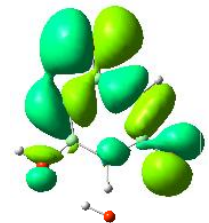

BA7

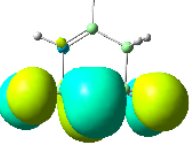

BA9

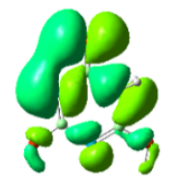

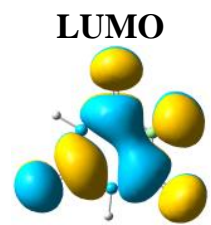
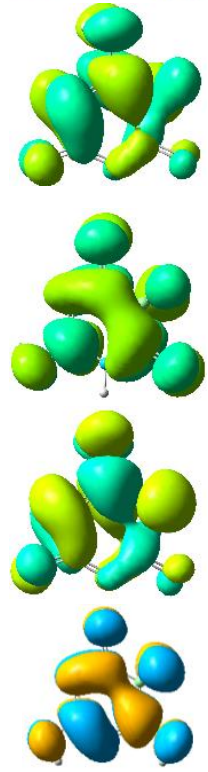
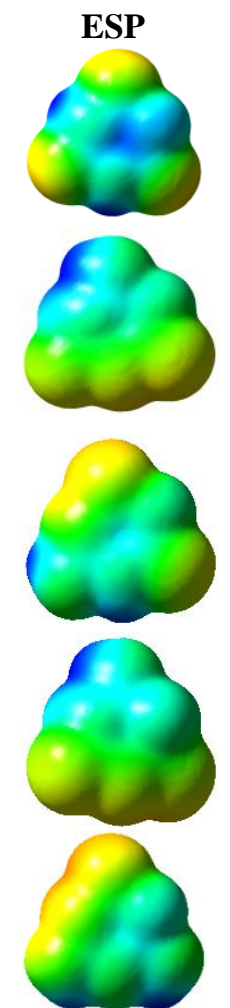

BA2

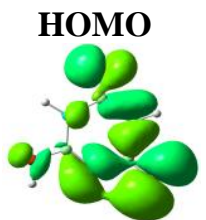

BA4

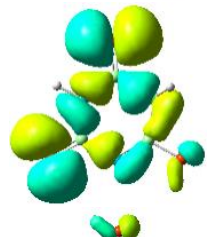

BA6
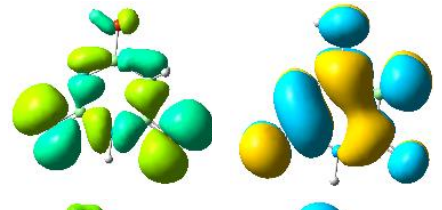

BA8

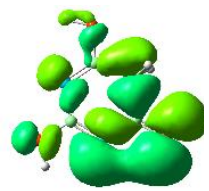

BA10

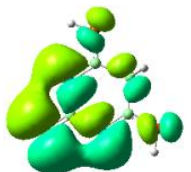

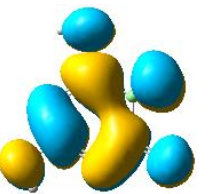
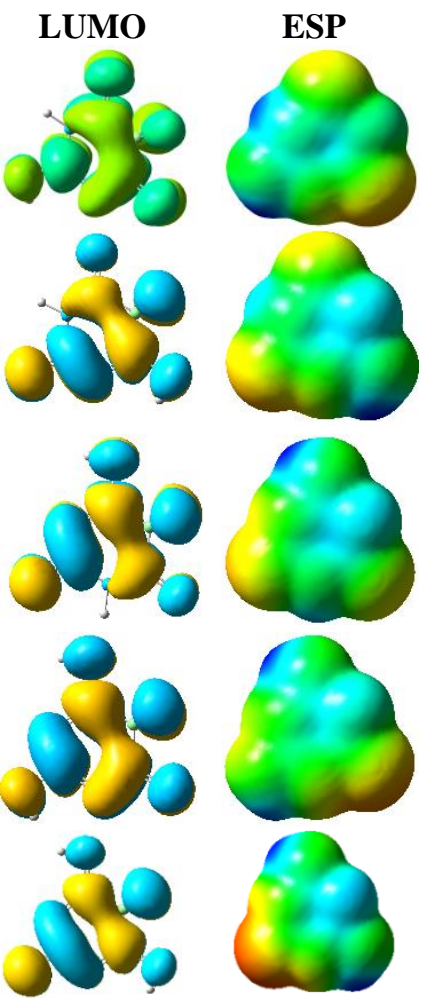

Figure 3. HOMO and LUMO distribution patterns and ESP surfaces for the models of BA.

Further analyses of this work were done using the evaluated quantities of Qcc for nitrogen, oxygen, and hydrogen atoms of BA1 - BA10 tautomers. The results were listed in Table 2, and the related diagram was also included for interpretation of such molecular systems at the atomic scales. Qcc originally arises from atoms' electronic site, and it could very well describe such a chemical environment $[39,40]$. To this benefit, such atomic-scale features were evaluated for the investigated models of this work. A quick look at the results of Table 2 could reveal that almost all atoms' chemical environments detected more or less significant effects of tautomerism compared with the original BA1 reference model. In such a case, those atoms directly dealing with the hydrogen atom movement could show many more significant effects of such perturbations. It is important to note that the values of Qcc for hydrogen atoms were very much small regarding their low density of electronics at the atomic sites, whereas those of oxygen atoms were at the largest values in contrast. In BA1, pure keto form was available with locating hydrogen atoms at the N1 and N4 atomic sites; however, in none of the other tautomers, such system was available. In BA2 - BA7, one atom of N1 or N4 had a hydrogen atom, but none of them had a hydrogen atom in BA8 - BA10. In such cases, changes in atomic environments could lead to changes in electronic features detectable by quantities of Qcc. The magnitude of such quantity could show how much electron density was accepted or donated in a molecular system. If an atom releases its electronic density, it will result in a lower value of Qcc. Consequently, changes of BA1 - BA10 tautomers' atomic-scale properties could warn for their careful use for specified applications in biological media. 
Table 2. Quadrupole coupling constants ( $\mathrm{MHz})$, atomic labeles and related diagram.

\begin{tabular}{c|c|c|c|c|c|c|c|c|c} 
Model & N1 & N4 & O3 & O6 & O9 & H10 & H11 & H12 & H13 \\
\hline BA1 & 3.759 & 3.759 & 8.755 & 9.582 & 9.582 & 0.193 & 0.193 & $0.254 \mathrm{~N} 1$ & $0.254 \mathrm{~N} 4$ \\
\hline BA2 & 3.005 & 3.834 & 9.024 & 9.633 & 10.458 & 0.196 & 0.191 & 0.289 O3 & $0.256 \mathrm{~N} 4$ \\
\hline BA3 & 3.068 & 1.658 & 7.641 & 9.105 & 9.814 & 0.195 & 0.191 & 0.294 O4 & $0.250 \mathrm{~N} 4$ \\
\hline BA4 & 3.471 & 3.568 & 9.718 & 9.412 & 8.751 & 0.193 & 0.192 & 0.287 O6 & $0.256 \mathrm{~N} 4$ \\
\hline BA5 & 3.834 & 3.005 & 9.026 & 10.457 & 9.634 & 0.196 & 0.191 & $0.256 \mathrm{~N} 1$ & $0.289 \mathrm{O} 3$ \\
\hline BA6 & 3.568 & 3.471 & 9.718 & 8.751 & 9.412 & 0.193 & 0.192 & $0.255 \mathrm{~N} 1$ & 0.287 O6 \\
\hline BA7 & 1.658 & 3.068 & 7.643 & 9.814 & 9.104 & 0.195 & 0.191 & $0.250 \mathrm{~N} 1$ & 0.294 O9 \\
\hline BA8 & 3.156 & 3.286 & 8.641 & 8.686 & 10.233 & 0.194 & 0.193 & 0.289 O3 & 0.286 O6 \\
\hline BA9 & 3.013 & 3.468 & 8.896 & 10.312 & 8.738 & 0.194 & 0.193 & 0.295 O3 & 0.289 O9 \\
\hline BA10 & 3.536 & 3.536 & 10.804 & 8.773 & 8.773 & 0.093 & 0.0931 & 0.160 O6 & 0.160 O9 \\
\hline
\end{tabular}
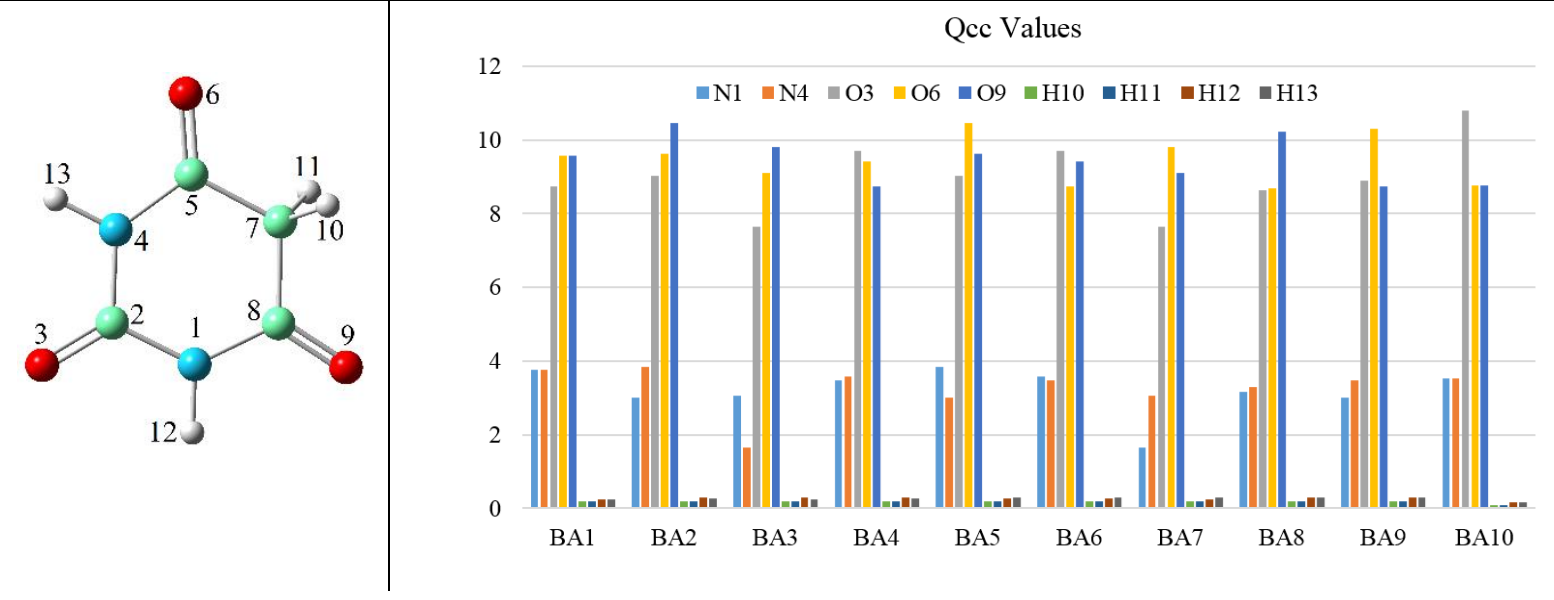

\section{Conclusions}

Within this work, tautomeric formations of BA were investigated using the computerbased DFT method to investigate the effects of such hydrogen movement processes on the molecular and atomic scales features of BA. In this regard, molecular and atomic descriptors were evaluated for ten tautomers' optimized structures; BA1 - BA10. The results indicated that the ring structure could be broken for two of tautomers BA3 and BA7, in which their stabilities were at the lowest level among other tautomers. Although such ring structure remained for other tautomers, further frontier molecular orbitals feature indicated meaningful effects for tautomeric structures' electronic properties. In this case, HOMO, LUMO, EG, FE, and even ESP showed the changes of such electronic environment for the tautomeric system more or less significant for them. Indeed, such variations are very much important for activities of structures, in which different activities from those already specified will be expected from such electronic variations. Further analyses based on atomic-scale Qcc values also indicated the changes in atoms' chemical environments at the lowest atomic scales. In this case, such results all determined the electronic variations of structures for pure keto to keto-enol tautomeric conversions warning for careful designing any related experiments before performing practically.

\section{Funding}

This research received no external funding.

\section{Acknowledgments}

This research has no acknowledgment. 


\section{Conflicts of Interest}

\section{The authors declare no conflict of interest.}

\section{References}

1. Freitag, F.; Wagner, E. Optimizing synthetic nucleic acid and protein nanocarriers: The chemical evolution approach. Advanced Drug Delivery Reviews 2021, 168, 30-54, https://doi.org/10.1016/j.addr.2020.03.005.

2. Su, Y.; Chu, H.; Tian, J.; Du, Z.; Xu, W. Insight into the nanomaterials enhancement mechanism of nucleic acid amplification reactions. TrAC Trends in Analytical Chemistry 2021, 137, https://doi.org/10.1016/j.trac.2021.116221.

3. Watson, J.D.; Crick, F.H. Molecular structure of nucleic acids: a structure for deoxyribose nucleic acid. Nature 1953, 171, 737-738, https://doi.org/10.1038/171737a0.

4. Fernández, R.; Gabaldón, T. Gene gain and loss across the metazoan tree of life. Nature Ecology \& Evolution 2020, 4, 524-533, https://doi.org/10.1038/s41559-019-1069-X.

5. Di Iorio, P.; Ciccarelli, R. Adenine-based purines and related metabolizing enzymes: evidence for their impact on tumor extracellular vesicle activities. Cells 2021, 10, https://doi.org/10.3390/cells10010188.

6. Stanojević, A.; Milovanović, B.; Stanković, I.; Etinski, M.; Petković, M. The significance of the metal cation in guanine-quartet-metalloporphyrin complexes. Physical Chemistry Chemical Physics 2021, 23, 574-584, https://doi.org/10.1039/D0CP05798C.

7. Mirzaei, M.; Elmi, F.; Hadipour, N.L. A systematic investigation of hydrogen-bonding effects on the 17O, $14 \mathrm{~N}$, and $2 \mathrm{H}$ nuclear quadrupole resonance parameters of anhydrous and monohydrated cytosine crystalline structures: a density functional theory study. The Journal of Physical Chemistry B 2006, 110, 10991-10996, https://doi.org/10.1021/jp060301g.

8. Mirzaei, M.; Kalhor, H.R.; Hadipour, N.L. Covalent hybridization of CNT by thymine and uracil: A computational study. Journal of Molecular Modeling 2011, 17, 695-699, https://doi.org/10.1007/s00894010-0771-z.

9. Milovanović, B.; Novak, J.; Etinski, M.; Domcke, W.; Došlić, N. Simulation of UV absorption spectra and relaxation dynamics of uracil and uracil-water clusters. Physical Chemistry Chemical Physics 2021, 23, 2594-2604, https://doi.org/10.1039/D0CP05618A.

10. Yang, H.; Eremeeva, E.; Abramov, M.; Herdewijn, P. The network of replication, transcription, and reverse transcription of a synthetic genetic cassette. Angewandte Chemie 2021, 133, 4221-4228, https://doi.org/10.1002/anie.202011887.

11. Li, J.; Wang, Z.; Hua, Z.; Tang, C. Supramolecular nucleobase-functionalized polymers: synthesis and potential biological applications. Journal of Materials Chemistry B 2020, 8, 1576-1588, https://doi.org/10.1039/C9TB02393C.

12. Han, J.; Funk, C.; Eyberg, J.; Bailer, S.; Richert, C. An AZT analog with strongly pairing ethynylpyridone nucleobase and its antiviral activity against HSV1. Chemistry \& Biodiversity 2021, 18, https://doi.org/10.1002/cbdv.202000937.

13. Faramarzi, R.; Falahati, M.; Mirzaei, M. Interactions of fluorouracil by CNT and BNNT: DFT analyses. Advanced Journal of Science and Engineering 2020, 1, 62-66, https://doi.org/10.22034/AJSE.2012062.

14. Yaghoobi, R.; Mirzaei, M. Computational analyses of cytidine and aza-cytidine molecular structures. Labin-Silico 2020, 1, 21-66.

15. Jubeen, F.; Liaqat, A.; Amjad, F.; Sultan, M.; Iqbal, S.Z.; Sajid, I.; Khan Niazi, M.B.; Sher, F. Synthesis of 5-fluorouracil cocrystals with novel organic acids as coformers and anticancer evaluation against HCT-116 colorectal cell lines. Crystal Growth \& Design 2020, 20, 2406-2414, https://doi.org/10.1021/acs.cgd.9b01570.

16. Gilani, A.G.; Taghvaei, V.; Rufchahi, E.M.; Mirzaei, M. Tautomerism, solvatochromism, preferential solvation, and density functional study of some heteroarylazo dyes. Journal of Molecular Liquids 2019, 273, 392-407, https://doi.org/10.1016/j.molliq.2018.10.054.

17. Kanakaveti, V.; Anoosha, P.; Sakthivel, R.; Rayala, S.K.; Gromiha, M.M. Quantitative structure-activity relationship in ligand based drug design: Concepts and applications. Protein Interactions 2020, 333-349, https://doi.org/10.1142/9789811211874_0013.

18. Zhang, J.; Qi, S.; Zhang, C.; Fan, Z.; Ding, Q.; Mao, S.; Dong, Z. Controlling keto-enol tautomerism of ureidopyrimidinone to generate a single-quadruple AADD-DDAA dimeric array. Organic Letters 2020, 22, 7305-7309, https://doi.org/10.1021/acs.orglett.0c02644.

19. Shahzad, H.; Ahmadi, R.; Najafpour, J.; Adhami, F. Adsorption of cytarabine on the surface of fullerene C20: a comprehensive DFT study. Eurasian Chemical Communications 2020, 2, 162-169, https://doi.org/10.33945/SAMI/ECC.2020.2.1.

20. Afshar, M.; Ranjineh Khojasteh, R.; Ahmadi, R. Adsorption of lomustin anticancer drug on the surface of carbon nanotube: a theoretical study. Eurasian Chemical Communications 2020, 2, 595-603, https://doi.org/10.33945/SAMI/ECC.2020.5.5. 
21. Mirzaei, M. Science and engineering in silico. Advanced Journal of Science and Engineering 2020, 1, 1-2, https://doi.org/10.22034/AJSE2011001.

22. Ozkendir, O.M. Electronic structure study of Sn-substituted InP semiconductor. Advanced Journal of Science and Engineering 2020, 1, 7-11, https://doi.org/10.22034/AJSE2011007.

23. Wang, Y.H.; Suk, F.M.; Liu, C.L.; Chen, T.L.; Twu, Y.C.; Hsu, M.H.; Liao, Y.J. Antifibrotic effects of a barbituric acid derivative on liver fibrosis by blocking the NF- $\kappa$ B signaling pathway in hepatic stellate cells. Frontiers in Pharmacology 2020, 11, https://doi.org/10.3389/fphar.2020.00388.

24. Ralhan, S.; Ray, N.K. Density functional study of barbituric acid and its tautomers. Journal of Molecular Structure: THEOCHEM 2003, 634, 83-88, https://doi.org/10.1016/S0166-1280(03)00260-4.

25. Schmidt, M.U.; Brüning, J.; Glinnemann, J.; Hützler, M.W.; Mörschel, P.; Ivashevskaya, S.N.; van de Streek, J.; Braga, D.; Maini, L.; Chierotti, M.R.; Gobetto, R. The thermodynamically stable form of solid barbituric acid: the enol tautomer. Angewandte Chemie 2011, 50, 7924-7926, https://doi.org/10.1002/anie.201101040.

26. Millefiori, S.; Millefiori, A. Tautomerism in barbituric and thiobarbituric acids. Journal of Heterocyclic Chemistry 1989, 26, 639-644, https://doi.org/10.1002/jhet.5570260324.

27. Okuno, M.; Yamada, S.; Ohto, T.; Tada, H.; Nakanishi, W.; Ariga, K.; Ishibashi, T.A. Hydrogen bonds and molecular orientations of supramolecular structure between barbituric acid and melamine derivative at the air/water interface revealed by heterodyne-detected vibrational sum frequency generation spectroscopy. The Journal of Physical Chemistry Letters 2020, 11, 2422-2429, https://doi.org/10.1021/acs.jpclett.0c00329.

28. Ding, S.; Yao, B.; Schobben, L.; Hong, Y. Barbituric acid based fluorogens: synthesis, aggregation-induced emission, and protein fibril detection. Molecules 2020, 25, https://doi.org/10.3390/molecules25010032.

29. Frisch, M.; Trucks, G.; Schlegel, H.; Scuseria, G.; Robb, M.; Cheeseman, J.; Montgomery Jr, J.; Vreven, T.; Kudin, K.; Burant, J. Gaussian 09 D.01 Program. Gaussian. Inc.: Wallingford, CT, USA. 2009.

30. Pence, H.E.; Williams, A. ChemSpider: an online chemical information resource. Journal of Chemical Education 2010, 87, 1123-1124, https://doi.org/10.1021/ed100697w.

31. Mirzaei, M.; Giahi, M. Computational studies on boron nitride and boron phosphide nanotubes: density functional calculations of boron-11 electric field gradient tensors. Physica E 2010, 42, 1667-1669, https://doi.org/10.1016/j.physe.2010.01.022.

32. Mirzaei, M.; Yousefi, M. Computational studies of the purine-functionalized graphene sheets. Superlattices and Microstructures 2012, 52, 612-617, https://doi.org/10.1016/j.spmi.2012.06.027.

33. Mirzaei, M.; Hadipour, N.L. Density functional calculations of $14 \mathrm{~N}$ and 11B NQR parameters in the Hcapped (6,0) and (4,4) single-walled BN nanotubes. Physica $E$ 2008, 40, 800-804, https://doi.org/10.1016/j.physe.2007.10.050.

34. Mirzaei, M.; Mirzaei, M. Sulfur doping at the tips of $(6,0)$ boron nitride nanotube: a DFT study. Physica E 2010, 42, 2147-2150, https://doi.org/10.1016/j.physe.2010.04.014.

35. Behzadi, H.; Hadipour, N.L.; Mirzaei, M. A density functional study of $17 \mathrm{O}, 14 \mathrm{~N}$ and $2 \mathrm{H}$ electric field gradient tensors in the real crystalline structure of $\alpha$-glycine. Biophysical Chemistry 2007, 125, 179-183, https://doi.org/10.1016/j.bpc.2006.07.010.

36. Trontelj, Z.; Pirnat, J.; Jazbinšek, V.; Lužnik, J.; Srčič, S.; Lavrič, Z.; Beguš, S.; Apih, T.; Žagar, V.; Seliger, J. Nuclear quadrupole resonance (NQR) - a useful spectroscopic tool in pharmacy for the study of polymorphism. Crystals 2020, 10, https://doi.org/10.3390/cryst10060450.

37. Stare, J.; Gradišek, A.; Seliger, J. Nuclear quadrupole resonance supported by periodic quantum calculations: a sensitive tool for precise structural characterization of short hydrogen bonds. Physical Chemistry Chemical Physics 2020, 22, 27681-27689, https://doi.org/10.1039/D0CP04710D.

38. Choudhary, K.; Ansari, J.N.; Mazin, I.I.; Sauer, K.L. Density functional theory-based electric field gradient database. Scientific Data 2020, 7, 1-10, https://doi.org/10.6084/m9.figshare.13027220.

39. Samadi, Z.; Mirzaei, M.; Hadipour, N.L.; Khorami, S.A. Density functional calculations of oxygen, nitrogen and hydrogen electric field gradient and chemical shielding tensors to study hydrogen bonding properties of peptide group (OC-NH) in crystalline acetamide. Journal of Molecular Graphics and Modelling 2008, 26, 977-981, https://doi.org/10.1016/j.jmgm.2007.08.003.

40. Mirzaei, M.; Gülseren, O.; Hadipour, N. DFT explorations of quadrupole coupling constants for planar 5fluorouracil pairs. Computational and Theoretical Chemistry 2016, 1090, 67-73, https://doi.org/10.1016/j.comptc.2016.06.004. 